\title{
Detection of spin torque magnetization dynamics through low frequency noise
}

\author{
Juan Pedro Cascales, ${ }^{1, a)}$ David Herranz, ${ }^{1}$ Ursula Ebels, ${ }^{2}$ Jordan A. Katine,${ }^{3}$ \\ and Farkhad G. Aliev ${ }^{1, b)}$ \\ ${ }^{1}$ Dpto. Fisica Materia Condensada C3, Instituto Nicolas Cabrera (INC), Condensed Matter Physics Institute \\ (IFIMAC), Universidad Autonoma de Madrid, Madrid 28049, Spain \\ ${ }^{2}$ SPINTEC, UMR 8191, CEA/CNRS/UJF \& G-INP, INAC, 38054 Grenoble Cedex, France \\ ${ }^{3}$ Hitachi Global Storage Technologies, San Jose, California 95135, USA
}

(Received 29 May 2015; accepted 23 July 2015; published online 3 August 2015)

\begin{abstract}
We present a comparative study of high frequency dynamics and low frequency noise in elliptical magnetic tunnel junctions with lateral dimensions under $100 \mathrm{~nm}$ presenting current-switching phenomena. The analysis of the high frequency oscillation modes with respect to the current reveals the onset of a steady-state precession regime for negative bias currents above $J=10^{7} \mathrm{~A} / \mathrm{cm}^{2}$, when the magnetic field is applied along the easy axis of magnetization. By the study of low frequency noise for the same samples, we demonstrate the direct link between changes in the oscillation modes with the applied current and the normalised low frequency (1/f) noise as a function of the bias current. These findings prove that low frequency noise studies could be a simple and powerful technique to investigate spin-torque based magnetization dynamics. ( 2015 AIP Publishing LLC.

[http://dx.doi.org/10.1063/1.4927827]
\end{abstract}

Oscillations known as a ferromagnetic resonance (FMR) arise from the precessional motion of the magnetization of a ferromagnetic material (FM) when an external magnetic field is applied in the presence of microwave pump field perpendicular to it. Since magnetic tunnel junctions (MTJs) are composed of ferromagnetic electrodes, and exhibit the tunneling magnetoresistance (TMR) effect, ${ }^{1-3}$ it is possible to detect magnetization dynamics through the measurement of frequency dependent voltage noise power (typically up to a few tens of GHz) in DC biased MTJs (see the review ${ }^{4}$ ). In the regime where the current density applied to the MTJ is low, the resulting damped oscillatory modes are due to the external applied magnetic field and thermal fluctuations, referred to as thermal FMR (T-FMR). This effect is typically observed for applied current densities below $J \simeq 10^{7} \mathrm{~A} / \mathrm{cm}^{2}{ }^{4}$ The effective damping can be cancelled altogether by the spin torque ${ }^{5}$ (ST) from a d.c., spin-polarized current at some critical value of the current density $J_{C}$. This results in an auto-oscillation of the magnetization which is often referred to as a steady state precession (SPP). The ability to switch the magnetic state of MTJs with only current could pave the way for smaller and faster data storage devices. Using MTJs with lateral sizes under $100 \mathrm{~nm}$ would increase the storage density of devices, reduce their power consumption, and contribute to the development of current controlled microwave sources.

The transition from the T-FMR regime to an in-plane, SSP can be identified from a sudden decrease in the frequency and linewidth $\Delta f$ on the applied current. ${ }^{6-10}$ The TFMR/SSP transition in fact presents two regimes with critical currents $J_{C}$ and $J_{C}^{*}$, where for $\left|J_{C}\right|<|J|<\left|J_{C}^{*}\right|$ the system presents an intermittent steady state (stable for a few ns) with linewidths in the hundreds of $\mathrm{MHz}$, which becomes

\footnotetext{
${ }^{\text {a)} E l e c t r o n i c ~ m a i l: ~ j u a n p e d r o . c a s c a l e s @ u a m . e s ~}$

b)Electronic mail: farkhad.aliev@uam.es
}

stable for several $\mu$ s when $\left|J_{C}^{*}\right|<|J|$ and presents linewidths an order of magnitude lower. ${ }^{11}$

Our work shows that MTJ samples with a magnetic field along the easy axis (EA) present spin-torque effects so the coercive field of the free electrode is shifted to lower values. Most importantly, the influence of changes with the current in the high frequency (HF) oscillation modes has also been observed through a systematic study of low frequency (LF) noise measurements. Previous studies indicate that the low frequency tail in the HF noise power could be affected by the transition from a damped oscillation to a steady state precession. ${ }^{12,13}$ The LF measurements presented here may constitute a better quantification of the stochastic hopping at the transition between the T-FMR to SSP in the $\mathrm{kHz}$ range. Also, this realization could be widely useful, as measurements in the $\mathrm{kHz}$ range are technically simpler than for high frequency signals (MHz-GHz).

The multilayer MTJ nanopillars have the following structure: $\operatorname{IrMn}(6.1) / \mathrm{CoFeB}(1.8) / \mathrm{Ru} / \mathrm{CoFe}(2) / \mathrm{MgO}(0.9 \mathrm{~nm}) /$ $\mathrm{CoFe}(0.5) / \mathrm{CoFeB}(3.4)$, where the numbers indicate the thickness of the layers in $\mathrm{nm}$. The pinned layer is part of a synthetic antiferromagnet (SAF) structure, consisting of two FM layers which are antiferromagnetically coupled through a thin ruthenium layer. The lower FM layer is exchangecoupled to an antiferromagnetic $\mathrm{IrMn}$ layer. The $\mathrm{MgO}$ barrier is deposited by sputtering, and the free layer consists of a bilayer of $\mathrm{CoFe} / \mathrm{CoFeB}$. The measured nanopillar devices have elliptical cross-sections of different sizes, with the minor and major axes ranging from $40 \times 80$ to $65 \times 130$ (in $\mathrm{nm}$ ), and a nominal resistance-area (RA) product of $1.5 \Omega \times \mu \mathrm{m}^{2}$. The EA direction is parallel to the pinned layer's magnetization coinciding with the major axis of the ellipse, while the inplane hard axis (HA) is perpendicular (but still in-plane) to the EA. The devices are embedded in impedance matched RF coplanar waveguides for electrical contacting using special 


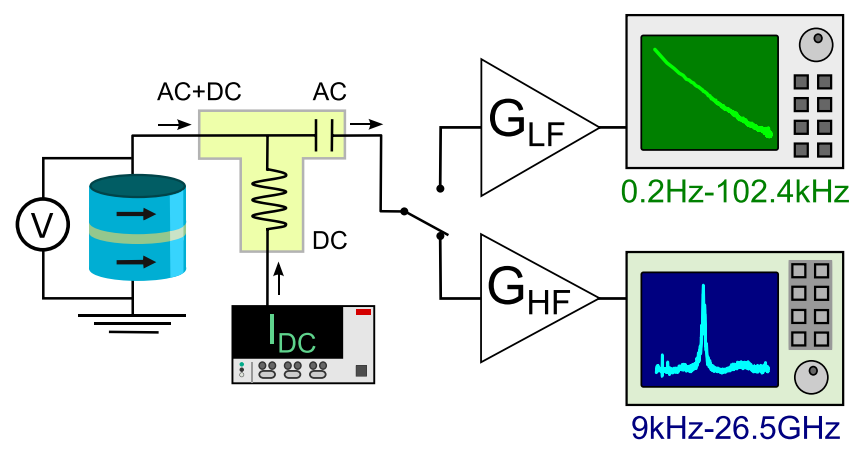

FIG. 1. Diagram of the experimental setup, where either a low ( $\mathrm{kHz}$ range) or high frequency (GHz range) noise measurement scheme can be selected.

RF probes. Figure 1 shows a diagram of the experimental setup. The samples are biased by a d.c. current which is input through the LF port of a bias tee, and the voltage across the device is measured by a nanovoltmeter. The voltage signal from the sample goes through the mixed port of the bias tee and is input into either LF or HF system through the HF port of the bias tee. The LF system was previously described in Ref. 14. Regarding the HF measurement, the voltage fluctuations out of the HF port are amplified by a Miteq AVG6 amplifier, and then input into an Agilent Technologies EXA signal analyzer (bandwidth $9 \mathrm{kHz}-26.5 \mathrm{GHz}$ ). Details of the calibration of the HF setup may be found in Ref. 15 .

Previous measurements on devices of this kind ${ }^{10,11,16}$ have shown that these MTJs fall into two different groups: samples with high resistance and TMR ratios around 90\% (labeled HTMR) and samples with low resistance and TMR ratios around 30\%-60\% (labeled LTMR). The authors report that repeated high-current measurements on HTMR devices may gradually turn them into LTMR devices. These LTMR devices seem to be stable against high-current measurements, and the authors speculate that the difference between sample types could be due to localized reductions in the tunneling barrier. ${ }^{10}$ Indeed, the statistics of our MTJs reveal a mean TMR value of around $60 \%$ and an average RA product of $1.8 \Omega \times \mu \mathrm{m}^{2}$ (in agreement with the nominal value of 1.5 $\Omega \times \mu \mathrm{m}^{2}$ ). MTJs with ultra-low RA are important for practical devices. For junctions with low $\mathrm{MgO}$ thicknesses (and low RA products), there exists a correlation between the TMR ratio and the MgO thickness. ${ }^{17} \mathrm{~A}$ decrease in TMR is experienced when the thickness of the $\mathrm{MgO}$ barrier is reduced locally (pseudo-pinholes). Obtaining MTJs with low RA products and high TMR ratios, for which homogeneous barriers are needed, are a real engineering challenge. The ST effect requires high current densities, and since $\mathrm{MgO}$ barriers only withstand a certain amount of voltage, having a low RA allows high currents to flow through the MTJ without causing the breakdown of the barrier. ${ }^{18} \mathrm{We}$ shall focus below on LTMR MTJs, as they are best candidates to study magnetization dynamics and spin torque effects.

Figure 2 shows that when the external field is directed along the easy axis, one observes a step-like transition in resistance, from a high resistance state at positive fields (antiparallel or AP state) to a lower resistance state at negative fields (parallel or $\mathrm{P}$ state). In our measurements, positive voltage means that electrons flow from the pinned to the free electrode, promoting the $\mathrm{P}$ state. Negative voltage favors the AP state. The representative high and low frequency noise measurements shown in this work were carried out in the same $40 \times 80 \mathrm{~nm}^{2}$ sample, although TMR, LF, and/or HF noise was measured in around 60 nanopillar MTJs. Fig. 2(a) shows changes in the coercive field with the applied current, obtained from resistive transitions measured by sweeping the field positive to negative values. A full TMR cycle taken at low bias $(2 \mathrm{mV})$ is shown in the inset of Fig. 2(a). As could be seen in Fig. 2(b), the coercive field $H_{C}$ is stable for negative currents (the AP state is favored), while it shifts to lower values (favoring the $\mathrm{P}$ state) for positive currents. Note that the results obtained from both high and low frequency setups have been plotted in Fig. 2(b).

A typical high frequency noise spectrum presents resonance peaks centered around a frequency $f_{\text {res }}$, with linewidths $\Delta f$. An example of such a spectrum is shown in Fig. 3(a). We have studied the evolution of these resonance modes with both an external magnetic field and a d.c. current $I$. The resonance peaks may be characterized by their $f_{\text {res }}$, $\Delta f$, and output power $P_{\text {out }}$ of the microwave emission. We have constructed surface plots (see Fig. 3(b)) at constant current, with the high frequency spectra taken at different applied external fields, so the evolution of the modes with the current can be detected. For positive currents, the P state is stabilized and the AP state is destabilized, and vice versa for negative currents. This is reflected in the fact that the modes observed have higher amplitudes in the AP state for positive currents, and in the $\mathrm{P}$ state for negative currents.

Six clear modes are detected, which come from oscillations of the free layer (labeled F0, F1, etc.) and the SAF structure (A0, A1, and A2). The free layer modes are Vshaped, while the modes not showing a minimum at low
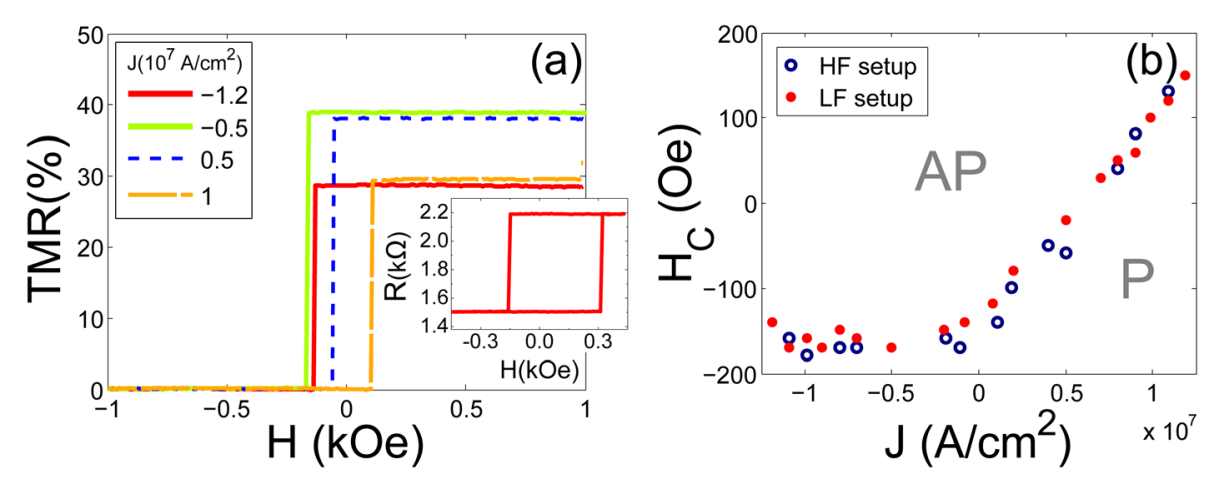

FIG. 2. $40 \times 80 \mathrm{~nm}^{2}$ MTJ biased along the easy axis direction. (a) TMR curves at different applied currents. The coercive field of the MTJ changes with increasing positive current. The inset shows a full TMR cycle. (b) Change in the coercive field with the applied current from HF and LF noise measurements. 

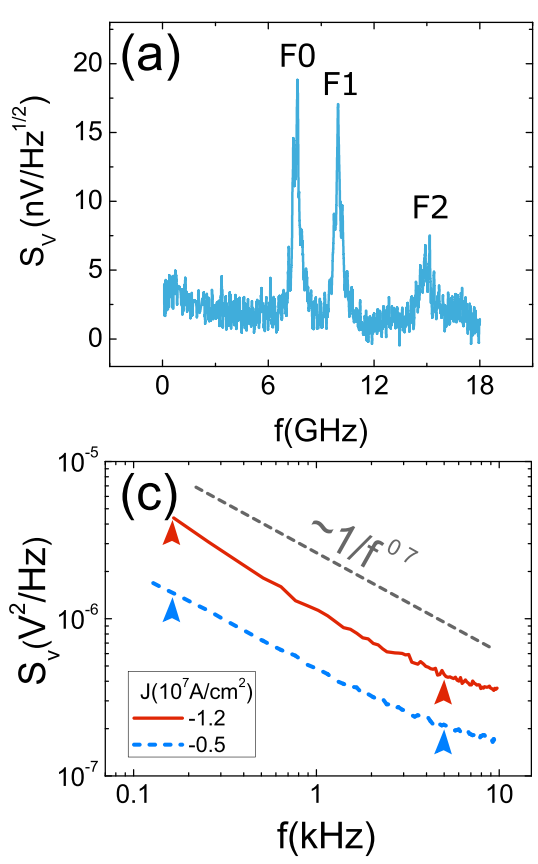
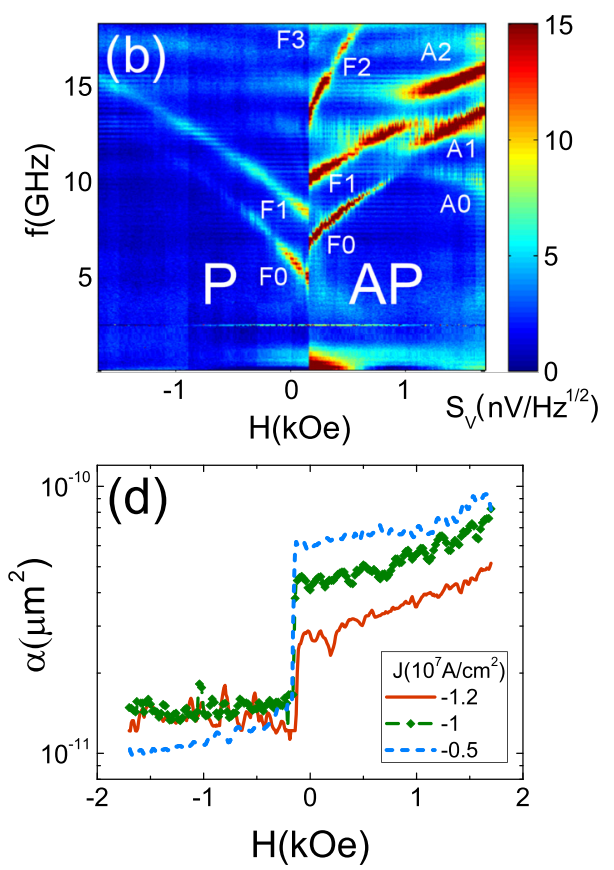

FIG. 3. (a) High frequency spectrum in the $\mathrm{P}$ state $(\mathrm{H}=-350 \mathrm{Oe})$ for $J=$ $-1.2 \cdot 10^{7} \mathrm{~A} / \mathrm{cm}^{2}$ of a $40 \times 80 \mathrm{~nm}$ elliptical MTJ. The spectrum reveals three different free layer oscillation modes, F0, F1, and F2. (b) Surface plots at $J=+1.2 \times 10^{7} \mathrm{~A} / \mathrm{cm}^{2}$ constructed with the high frequency spectra, presenting several oscillation modes of the FM layers. (c) Noise power spectra in the $\mathrm{P}$ state $(\mathrm{H}=-350 \mathrm{Oe})$, where the dashed line depicts a $\sim 1 / f^{0.7}$ dependence and (d) dependence of the Hooge parameter on the external field for several current density values. fields correspond to SAF modes. ${ }^{19}$ The SAF modes should present a minimum at the high field required for the spin-flop of the SAF, but our applied fields are not high enough. The F0 mode typically corresponds to excitations localized near the edges of the layer. ${ }^{19}$ The F2 mode only appears in the state which is excited ( $\mathrm{P}$ or AP), depending on the polarity of the current. A possible fourth free layer mode, F3, is labeled, although it appears very tenuous and is only present for $J=+1.2 \cdot 10^{7} \mathrm{~A} / \mathrm{cm}^{2}$. Several other samples revealed similar oscillation modes. We have carried out an analysis of the F0 and F1 modes (as they have the highest amplitudes) in the manner discussed in Ref. 10. The analysis of these results reveals that for negative currents, a decrease in frequency is observed for the first and second modes in the P state, starting at $J \sim-10^{7} \mathrm{~A} / \mathrm{cm}^{2}$. Under the same conditions, the AP state data presents the same dependence as the $\mathrm{P}$ state, but the features are not as clear. The change of the oscillation frequency with respect to the T-FMR regime and the linewidth of the $\mathrm{F} 0$ and $\mathrm{F} 1$ modes for the $\mathrm{P}(\mathrm{H}=-350 \mathrm{Oe})$ and AP (250 Oe) states is shown in Figs. 4(a) and 4(b). As can be seen, the decrease in frequency of the F0 and F1 modes in the $\mathrm{P}$ state resembles the transition from T-FMR to SSP reported on similar devices in Ref. 10. The minimum linewidth $\Delta f$ obtained for the $\mathrm{FO}$ mode is around $400 \mathrm{MHz}$, which agrees with what was previously observed for the intermittent steady state in Ref. 11. The microwave power was found to monotonically increase with the current and is not shown for brevity. Therefore, we conclude that our highest negative current takes our sample to an intermittent SSP regime. ${ }^{11}$ The AP state, however, presents no signs of such a transition, and the frequency of the modes in Fig. 4(a) remains constant with the current. Positive currents (not shown) also do not show such features in either magnetic state.

Low frequency noise measurements were also carried out in the same sample, using the same current and magnetic field values. The low frequency spectra (see Fig. 3(c)) may be described by $S_{V}(f)=\frac{\alpha V^{2}}{A f^{\beta}}$, where $V$ is the applied voltage,
$A$ is the sample area, $\alpha$ is the normalized $1 / \mathrm{f}$ noise or Hooge parameter, and $0.7<\beta<1.5$. The extraction of the $1 / \mathrm{f}$ parameters was carried out by carrying out a linear fit to $\log \left(S_{V}\right)=\log \left(\alpha V^{2} / A\right)-\beta \log (f)$ between $0<f<5 \mathrm{kHz}$, yielding a $\beta$ value of 0.7 on average. The spectra reveal the appearance of random telegraph noise at higher frequencies. ${ }^{14}$ The Hooge parameter remains somewhat constant for each magnetic state (P or AP) (see Fig. 3(d)), and it is higher in the AP state than for the P state because the sample presents resistance fluctuations which are proportional to the magnetization noise and $\partial R(H) / \partial H .^{20}$ The Hooge values obtained around $10^{-11} \mu \mathrm{m}^{2}$ reflect the high structural quality of the samples when compared to what was obtained for epitaxial MTJs with improved $\mathrm{Fe} / \mathrm{MgO}$ interfaces $\left(\sim 10^{-9}\right.$ $\left.\mu \mathrm{m}^{2}\right){ }^{21}$ The analysis of the $1 / \mathrm{f}$ noise data as a function of the current density reveals the signature of effects observed in

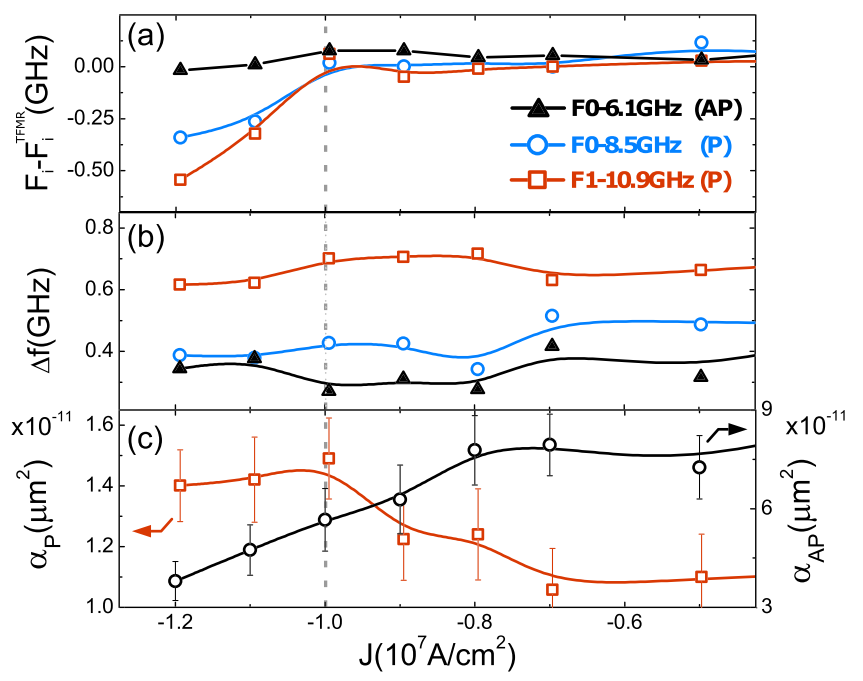

FIG. 4. High and low frequency noise measurements in the $\mathrm{P}(-350 \mathrm{Oe})$ and AP (250 Oe) states of a $40 \times 80 \mathrm{~nm}^{2}$ MTJ. Dependence of the F0 and F1 modes' (a) frequency shift with respect to the frequency in the T-FMR regime, (b) linewidth, and (c) average Hooge parameter as a function of the current density in the $\mathrm{P}$ and AP states. 
the HF results. As is shown in Fig. 4(c), noise in the P state, $\alpha_{P}$, monotonically increases with the applied current for current densities substantially below $\pm 10^{7} \mathrm{~A} / \mathrm{cm}^{2}$. This trend is observed regardless of the field range within the $\mathrm{P}$ state chosen for the average. Only for negative currents, the normalized noise reaches a maximum at around $J=-10^{7} \mathrm{~A} / \mathrm{cm}^{2}$.

$1 / \mathrm{f}$ noise in spin torque oscillators has been tied to hopping of the oscillation modes, where each random hopping event leads to a jump in the phase of the oscillator. ${ }^{22,23}$ As the current is increased towards the critical value for an intermittent $\operatorname{SSP}^{11}\left(J_{C}=-10^{7} \mathrm{~A} / \mathrm{cm}^{2}\right)$, these phase jumps increase in number, which is reflected in $1 / \mathrm{f}$ results. When the intermittent SSP is reached, the hopping events gradually become less frequent until the purely SSP is reached for a second critical current ${ }^{11} J_{C}^{*}$ (not reached in our experiment). The increase in $1 / \mathrm{f}$ noise is observed only in the P state, as negative currents destabilize this state. Therefore, by comparing our LF and HF results, we conclude that we are detecting signs of spin-torque related phenomena in low frequency $1 / \mathrm{f}$ measurements. Further, the LF results seem to be more sensible to ST-driven effects than HF measurements, since the $1 / \mathrm{f}$ noise begins increasing for lower current densities than the ones needed to observe any change in the oscillation modes (T-FMR-SSP transition). We remark that qualitatively different LF noise was observed in HTMR junctions with spin torque effects being suppressed in the current range under study. These MTJs expected to have a more uniform barrier revealed a decrease in $\alpha$ with an increasing applied bias, similarly to what was previously observed for $\mathrm{Fe} / \mathrm{MgO} / \mathrm{Fe}$ MTJs with $2-3 \mathrm{~nm}$ thick $\mathrm{MgO}$ barriers. ${ }^{24-26}$

In summary, current switching effects have been observed in low TMR nanopillar MTJs of sizes under $100 \mathrm{~nm}$ (Refs. 10, 11 and 16) if the MTJs are biased along the easy axis, where an AP/P switch is favored for positive currents. The analysis of the high frequency oscillation modes with respect to the bias current reveals the onset of a steady-state precession regime for negative currents, when the field is applied along the easy axis. A comparison of this analysis with $1 / \mathrm{f}$ noise as a function of the current shows that the changes in magnetization dynamics in the $\mathrm{GHz}$ range are reflected in the low frequency noise. The beginning of the transition to the steady state regime appears as a maximum in the normalized 1/f noise (Hooge parameter). The obtained results should help to define the "current window range" for the potential application of nm sized magnetic tunnel junctions by using LF noise measurement techniques.
We would like to acknowledge the support by the Spanish MINECO (MAT2012-32743), and the Comunidad de Madrid through NANOFRONTMAG-CM (S2013/MIT2850) and CCC-UAM (SVORTEX).

${ }^{1}$ M. Julliere, Phys. Lett. A 54, 225 (1975).

${ }^{2}$ J. S. Moodera, L. R. Kinder, T. M. Wong, and R. Meservey, Phys. Rev. Lett. 74, 3273 (1995).

${ }^{3}$ T. Miyazaki and N. Tezuka, J. Magn. Magn. Mater. 139(3), L231-L234 (1995).

${ }^{4}$ D. Ralph and M. Stiles, J. Magn. Magn. Mater. 320, 1190 (2008).

${ }^{5}$ J. Slonczewski, J. Magn. Magn. Mater. 159(1), L1-L7 (1996).

${ }^{6}$ J.-V. Kim, Q. Mistral, C. Chappert, V. S. Tiberkevich, and A. N. Slavin, Phys. Rev. Lett. 100, 167201 (2008).

${ }^{7}$ V. Tiberkevich, A. Slavin, and J.-V. Kim, Appl. Phys. Lett. 91, 192506 (2007).

${ }^{8}$ Q. Mistral, J.-V. Kim, T. Devolder, P. Crozat, C. Chappert, J. A. Katine, M. J. Carey, and K. Ito, Appl. Phys. Lett. 88, 192507 (2006).

${ }^{9}$ S. Petit, C. Baraduc, C. Thirion, U. Ebels, Y. Liu, M. Li, P. Wang, and B. Dieny, Phys. Rev. Lett. 98, 077203 (2007).

${ }^{10}$ D. Houssameddine, S. H. Florez, J. A. Katine, J.-P. Michel, U. Ebels, D. Mauri, O. Ozatay, B. Delaet, B. Viala, L. Folks et al., Appl. Phys. Lett. 93, 022505 (2008).

${ }^{11}$ D. Houssameddine, U. Ebels, B. Dieny, K. Garello, J.-P. Michel, B. Delaet, B. Viala, M.-C. Cyrille, J. A. Katine, and D. Mauri, Phys. Rev. Lett. 102, 257202 (2009).

${ }^{12}$ T. Devolder, L. Bianchini, J.-V. Kim, P. Crozat, C. Chappert, S. Cornelissen, M. Op de Beeck, and L. Lagae, J. Appl. Phys. 106, 103921 (2009).

${ }^{13}$ K. V. Thadani, G. Finocchio, Z.-P. Li, O. Ozatay, J. C. Sankey, I. N. Krivorotov, Y.-T. Cui, R. A. Buhrman, and D. C. Ralph, Phys. Rev. B 78, 024409 (2008).

${ }^{14}$ J. P. Cascales, D. Herranz, J. L. Sambricio, U. Ebels, J. A. Katine, and F. G. Aliev, Appl. Phys. Lett. 102, 092404 (2013).

${ }^{15}$ J. P. Cascales, Ph.D. dissertation, Universidad Autónoma de Madrid, Spain, 2015.

${ }^{16}$ D. Houssameddine, Ph.D. thesis, Université Joseph-Fourier - Grenoble I, 2009.

${ }^{17}$ S. Yuasa, T. Nagahama, A. Fukushima, Y. Suzuki, and K. Ando, Nat. Mater. 3, 868 (2004).

${ }^{18}$ J. Alvarez-Hérault, Ph.D. thesis, Université de Grenoble, 2010.

${ }^{19}$ A. Helmer, S. Cornelissen, T. Devolder, J.-V. Kim, W. van Roy, L. Lagae, and C. Chappert, Phys. Rev. B 81, 094416 (2010).

${ }^{20}$ H. T. Hardner, M. B. Weissman, M. B. Salamon, and S. S. P. Parkin, Phys. Rev. B 48, 16156 (1993).

${ }^{21}$ D. Herranz, F. Bonell, A. Gomez-Ibarlucea, S. Andrieu, F. Montaigne, R. Villar, C. Tiusan, and F. G. Aliev, Appl. Phys. Lett. 96, 202501 (2010).

${ }^{22}$ A. Eklund, S. Bonetti, S. R. Sani, S. Majid Mohseni, J. Persson, S. Chung, S. Amir Hossein Banuazizi, E. Iacocca, M. Ostling, J. Akerman et al., Appl. Phys. Lett. 104, 092405 (2014).

${ }^{23}$ R. Sharma, P. Drrenfeld, E. Iacocca, O. G. Heinonen, J. Kerman, and P. K. Muduli, Appl. Phys. Lett. 105, 132404 (2014).

${ }^{24}$ A. Gokce, E. R. Nowak, S. H. Yang, and S. S. P. Parkin, J. Appl. Phys. 99, 08A906 (2006).

${ }^{25}$ F. G. Aliev, R. Guerrero, D. Herranz, R. Villar, F. Greullet, C. Tiusan, and M. Hehn, Appl. Phys. Lett. 91, 232504 (2007).

${ }^{26}$ J. Almeida, P. Wisniowski, and P. Freitas, IEEE Trans. Magn. 44, 2569-2572 (2008). 